ISSN 1980-5098

\title{
BIOLOGIA DE Thaumastocoris peregrinus CARPINTERO E DELLAPÉ (HEMIPTERA: THAUMASTOCORIDAE) EM DEZ ESPÉCIES DE EUCALIPTO
}

\author{
BIOLOGY OF Thaumastocoris peregrinus CARPINTERO AND DELLAPÉ \\ (HEMIPTERA: THAUMASTOCORIDAE) IN TEN SPECIES OF EUCALYPTUS
}

Márcia Aparecida Smaniotto ${ }^{1}$ Alexandre da Silva ${ }^{2}$ Uemerson Silva da Cunha ${ }^{3}$ Mauro Silveira Garcia ${ }^{3}$

\section{RESUMO}

O percevejo-bronzeado-do-eucalipto Thaumastocoris peregrinus é originário da Austrália e foi detectado no Brasil em 2008, causando danos em plantações de eucalipto. O percevejo suga a seiva, principalmente das folhas mais velhas, nas quais se formam pontos cloróticos que, posteriormente, tornam-se manchas bronzeadas. Em casos extremos pode ocorrer desfolhação. Os trabalhos sobre o comportamento do inseto ainda são escassos perante os registros de danos em plantações de eucalipto. Diante disso, avaliou-se a biologia de Thaumastocoris peregrinus em dez espécies de eucalipto, plantadas comercialmente no Rio Grande do Sul. Das espécies oferecidas para alimentação de Thaumastocoris peregrinus, Eucalyptus dunnii foi a que conferiu maior longevidade, número de adultos, ovos e viabilidade aos insetos.

Palavras-chave: espécie-praga florestal; Eucalyptus spp.; percevejo-bronzeado-do-eucalipto.

\begin{abstract}
The bronze bug Thaumastocoris peregrinus is native from Australia and has been detected in Brazil since 2008, causing damages in eucalyptus plantations. This insect feeds on phloem-sap, preferentially on the oldest leaves that evolves into chlorotic spots and later in bronze spots. In high infestations, plant defoliation may occur. Although the presence of bronze bug on eucalyptus trees has been often recorded, still there is lack of behavioral studies. Therefore, the biology of the Thaumastocoris peregrinus was evaluated in different species of eucalyptus, commercially available in Rio Grande do Sul state. From all tested species, Eucalyptus dunnii conferred the greatest longevity, number of adults, egg and insect viability.
\end{abstract}

Keywords: forest pests; Eucalyptus spp.; bug bronze.

\section{INTRODUÇÃO}

O monocultivo do eucalipto, em grandes áreas, tem favorecido o aparecimento de insetos-praga (GOULD, 1998). Dentre as pragas do eucalipto, muitas possuem a mesma origem do seu hospedeiro, sendo nativas principalmente da Austrália (WINGFIELD et al., 2008).

Nesse contexto, o percevejo-bronzeado-do-eucalipto foi diagnosticado no Brasil em 2008 e tem

1 Engenheira Agrônoma, Dra ., Professora do Instituto Federal de Educação Ciência e Tecnologia do Rio Grande do Sul (IFRS), Campus Sertão, Rodovia RS 135, Km 25, Distrito Eng. Luiz Englert, CEP 99170-000, Sertão (RS), Brasil.marcia.smaniotto@sertao.ifrs.edu.br

2 Engenheiro Agrônomo, MSc., Rua Paissandu 234, CEP 99010-100, Passo Fundo (RS), Brasil. alexandresilva4@hotmail.com

3 Engenheiro Agrônomo, Dr., Professor do Departamento de Fitossanidade, Faculdade de Agronomia Eliseu Maciel, Universidade Federal de Pelotas, Caixa Postal 354, CEP 96160-000, Pelotas (RS), Brasil. uscunha@yahoo.com.br / garciasmauro@yahoo.com.br 
causado danos nesses plantios. Este inseto pertence ao gênero Thaumastocoris que possui mais de dez espécies descritas. A descrição taxonômica de Thaumastocoris peregrinus foi realizada por Carpintero e Dellapé em 2006. Na Itália, Thaumastocoris peregrinus foi relatado infestando Eucalyptus spp., sendo o primeiro registro de um membro da família Thaumastocoridae na Europa e região do Mediterrâneo (LAUDONIA; SASSO, 2012).

Insetos pertencentes à família Thaumastocoridae são pequenos percevejos fitófagos, sendo que o percevejo-bronzeado-do-eucalipto apresenta corpo achatado, com aproximadamente três milímetros de comprimento, placas mandibulares desenvolvidas, antenas com quatro segmentos e olhos avermelhados. Os adultos apresentam coloração marrom-clara com áreas mais escuras. Consiste em uma espécie fitófaga no estágio ninfal e adulto (CARPINTERO; DELLAPÉ, 2006), ágil, com apêndices apicais nas tíbias, o que lhes confere a capacidade de agarrar-se em superfícies lisas (JACOBS; NESER, 2005). Apresentam ciclo de vida (ovo-adulto) de aproximadamente 50 dias, podendo variar com a temperatura. Cada fêmea oviposita em média 60 ovos, de coloração preta e que são depositados de forma agregada, principalmente em superfícies irregulares. Seus danos são caracterizados pela sucção de seiva e consequente redução de área fotossintética da folha, que leva à desfolha parcial ou total e até mesmo à morte das árvores (BUTTON, 2007).

Diante disso, desenvolveu-se um estudo com o objetivo de descrever a biologia de Thaumastocoris peregrinus em dez espécies de eucalipto plantadas no Rio Grande do Sul.

\section{MATERIAIS E MÉTODOS}

O trabalho foi desenvolvido no laboratório de Biologia de Insetos e Controle Biológico do Departamento de Fitossanidade, da Faculdade de Agronomia "Eliseu Maciel", da Universidade Federal de Pelotas - UFPel, localizado no município de Capão do Leão - RS.

Inicialmente, foram coletados ovos, ninfas e adultos de Thaumastocoris peregrinus no campo, em plantio comercial, e foi estabelecida a criação de manutenção em laboratório. A incubação ocorreu em placas de petri e, à medida que as ninfas foram eclodindo, foram incorporadas na criação. Os adultos e ninfas foram mantidos em ramos de Eucalyptus dunnii, por ser a espécie de eucalipto com maior infestação pelo inseto no campo. Os ramos foram inseridos em um recipiente contendo água, distribuídos em gaiolas de vidro cobertas com voil, em temperatura ambiente $\left(26 \pm 2^{\circ} \mathrm{C}\right)$, umidade relativa de $65 \% \pm 10$, fotofase de 13 horas.

Foram testadas dez espécies de eucalipto que eram utilizadas para avaliar a adaptação ao Sul do Rio Grande do Sul, visando à produção de celulose, sendo: Eucalyptus badjensis, Eucalyptus benthamii, Eucalyptus bicostata, Eucalyptus dorrigoensis, Eucalyptus dunnii, Eucalyptus globulus, Eucalyptus grandis, Eucalyptus saligna, Eucalyptus smithii e Eucalyptus viminallis. Como o inseto prefere alimentarse de folhas maduras de eucalipto, ramos que apresentavam folhas nessas condições foram coletados nos plantios e levados ao laboratório, no qual permaneceram por um período máximo de três dias, quando eram substituídos por outros recém-colhidos.

Cada espécie de eucalipto contou com 25 repetições, nas quais todos os insetos permaneceram em confinamento e avaliou-se o estágio ninfal, número de instar, período reprodutivo, fecundidade, viabilidade de ovos, período embrionário, longevidade e ciclo biológico (ovo-adulto). As médias foram comparadas através do teste qui-quadrado e, após, empregou-se o teste Tukey a $5 \%$ de probabilidade. As análises foram realizadas utilizando-se o SAS (SAS INSTITUTE, 2002).

\section{RESULTADOS E DISCUSSÃO}

\section{Estágio ninfal e número de instar}

A duração média do primeiro instar variou entre as espécies, sendo mais rápida em Eucalyptus

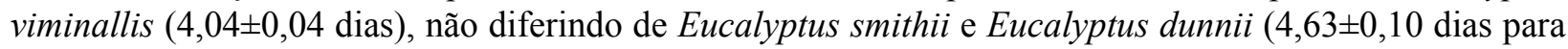
ambas). Nas demais espécies, não houve diferença. O número de ninfas reduziu significativamente em Eucalyptus bicostata e Eucalyptus globulus durante o primeiro instar. O segundo instar foi reduzido em 


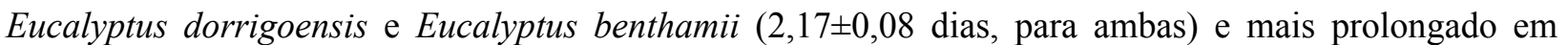

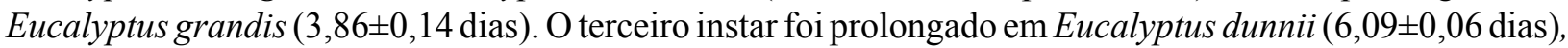
diferindo significativamente de todas as outras espécies, sendo reduzido em Eucalyptus smithii $(2,91 \pm 0,17$ dias) e Eucalyptus viminalis (2,95 0,15 dias). Já no quarto instar, o desenvolvimento foi extremamente rápido em Eucalyptus dunnii, sendo de apenas dois dias, diferindo das demais espécies. No quinto estádio de desenvolvimento houve variações entre as espécies, sendo menor em Eucalyptus bicostata $(4,00 \pm 0,58$ dias) e mais longo em Eucalyptus dunnii (5,91 $\pm 0,06$ dias). Soliman et al. (2012) relatam que as maiores durações médias do quinto instar de Thaumastocoris peregrinus foram obtidas em Eucalyptus grandis e Eucalyptus camaldulensis.

A duração média do primeiro e último instares foi superior àquelas encontradas nos instares intermediários. Esses resultados corroboram aqueles encontrados por Noack e Rose (2007) e por Soliman et al. (2012), em Eucalyptus scoparia. A duração total de desenvolvimento, desde a eclosão da ninfa até a fase adulta foi menor em Eucalyptus benthamii. Essa mesma quantidade de instares foi relatada para outros Thaumastocoridae, como Discocoris drakei Slater e Ashlock (COUTURIER et al., 2002).

O percevejo-bronzeado Thaumastocoris peregrinus apresentou um total de cinco instares, para nove das dez espécies de eucaliptos estudados, pois em Eucalyptus globulus apenas o primeiro instar pode ser acompanhado, sendo que ao final deste, a mortalidade de ninfas chegou a $100 \%$. Dentre as espécies estudadas, os insetos que se alimentaram em Eucalyptus globulus e Eucalyptus bicostata tiveram o desenvolvimento prejudicado, possivelmente em função da cerosidade presente nas folhas dessas duas espécies de eucalipto. Para Eucalyptus bicostata, assim como Eucalyptus globulus, apenas quatro insetos chegaram à fase adulta, demonstrando que essas espécies são inadequadas para o desenvolvimento de Thaumastocoris peregrinus.

\section{Período reprodutivo e fecundidade}

O início da oviposição variou entre as espécies estudadas, sendo que em Eucalyptus benthamii, dois dias após chegarem à fase adulta, as fêmeas iniciaram a oviposição, enquanto que nas demais a oviposição variou entre o quinto e o sétimo dia. O número de ovos/dia, em todas as espécies de eucaliptos estudadas, foi maior no início das avaliações e menor com o passar do tempo. O maior número de ovos/fêmea/dia foi de oito em Eucalyptus dunnii e Eucalyptus grandis, seguido de sete em Eucalyptus smithii. Estudos anteriores demonstraram taxas de oviposição mais elevados sobre Eucalyptus grandis do que em Eucalyptus camaldulensis (SOLIMAN et al., 2012). Assim, as espécies de eucalipto apresentam um impacto sobre as taxas de oviposição de Thaumastocoris peregrinus e, portanto, este é um importante fator quando se busca selecionar espécies para plantio em escala comercial.

Comparando-se o número total de ovos em folhas de diferentes espécies de eucalipto, observouse que o comportamento de oviposição de Thaumastocoris peregrinus sugere que Eucalyptus badjensis apresenta resistência do tipo antibiose, podendo estar associada a antixenose à oviposição (Tabela 1).

A razão sexual variou de acordo com as espécies de eucalipto estudadas. Somente Eucalyptus dorrigoensis apresentou proporção 1:1 (macho:fêmea) (Tabela 1).

\section{Viabilidade dos ovos}

A viabilidade de ovos de Thaumastocoris peregrinus foi superior a $94 \%$ na maioria das espécies de eucalipto estudadas, sendo a menor em Eucalyptus badjensis, com apenas 33,33\%, diferindo das demais espécies (Tabela 1). Os possíveis fatores que causaram estas taxas baixas de viabilidade dos ovos podem estar relacionados aos compostos secundários das plantas. Assim, Eucalyptus badjensis expressa algum tipo de resistência ao percevejo-bronzeado-do-eucalipto, apresentando compostos que podem ser tóxicos ao inseto ou inibidores de crescimento. Além disso, como não foram realizados testes com chance de escolha e com base nos resultados do estudo, é possível que no campo o inseto não iria optar por ovipositar em Eucalyptus badjensis. Estes resultados mostram o potencial dessa espécie de eucalipto quando indicada para o manejo do percevejo-bronzeado. 
TABELA 1: Número total de ovos por fêmea, período de oviposição (em dias), viabilidade dos ovos, razão sexual e período médio de incubação dos ovos de Thaumastocoris peregrinus (Hemiptera: Thaumastocoridae) criados em diferentes espécies de eucalipto (Temperatura $26 \pm 2^{\circ} \mathrm{C}$, UR de $60 \pm 10 \%$ e escotofase de 11 horas), Pelotas - RS, 2011. (Média seguida do erro padrão).

TABLE 1: Total number of eggs per female, oviposition period (in days), egg viability, sex ratio and average incubation period of eggs of Thaumastocoris peregrinus (Hemiptera: Thaumastocoridae) reared on different species of Eucalyptus (temperature $26 \pm 2^{\circ} \mathrm{C}$, RH $60 \pm 10 \%$ and scotophase 11 hours), Pelotas, RS state, 2011. (Average followed by the standard error).

\begin{tabular}{lccccc}
\hline \multirow{2}{*}{$\begin{array}{l}\text { Espécie } \\
\text { Total de ovos/ fêmea }\end{array}$} & \multicolumn{5}{c}{ Variável } \\
\cline { 2 - 6 } & $\begin{array}{c}\text { Período de } \\
\text { oviposição }\end{array}$ & $\begin{array}{c}\text { Viabilidade dos } \\
\text { ovos (\%) }\end{array}$ & Razão sexual & Período de incubação \\
\hline Eucalyptus badjensis & $01,00 \pm 0,00 \mathrm{c}$ & $01,00 \pm 0,00 \mathrm{c}$ & $33,33 \pm 33,33 \mathrm{~b}$ & 0,67 & 7 \\
Eucalyptus benthamii & $12,38 \pm 2,03 \mathrm{bc}$ & $05,13 \pm 0,81 \mathrm{bc}$ & $94,98 \pm 2,72 \mathrm{a}$ & 0,36 & 6 \\
Eucalyptus dorrigoensis & $05,89 \pm 0,87 \mathrm{c}$ & $03,00 \pm 0,44 \mathrm{c}$ & $73,08 \pm 6,48 \mathrm{a}$ & 0,50 & 6 \\
Eucalyptus dunnii & $53,90 \pm 3,59 \mathrm{a}$ & $17,30 \pm 1,05 \mathrm{a}$ & $97,65 \pm 0,63 \mathrm{a}$ & 0,45 & 4 \\
Eucalyptus grandis & $17,50 \pm 4,25 \mathrm{bc}$ & $05,50 \pm 0,68 \mathrm{bc}$ & $95,16 \pm 2,20 \mathrm{a}$ & - & 5 \\
Eucalyptus saligna & $03,17 \pm 0,75 \mathrm{c}$ & $02,00 \pm 0,37 \mathrm{c}$ & $96,67 \pm 3,33 \mathrm{a}$ & 0,43 & 7 \\
$\begin{array}{l}\text { Eucalyptus smithii } \\
\text { Eucalyptus viminalis }\end{array}$ & $16,56 \pm 5,49 \mathrm{bc}$ & $05,22 \pm 1,30 \mathrm{bc}$ & $94,27 \pm 2,54 \mathrm{a}$ & 0,48 & 5 \\
Coeficiente de & $27,67 \pm 4,70 \mathrm{~b}$ & $09,56 \pm 0,99 \mathrm{~b}$ & $96,26 \pm 1,21 \mathrm{a}$ & 0,40 & 5 \\
variação (\%) & 53,24 & 37,62 & 16,17 & & \\
\hline
\end{tabular}

Médias seguidas pela mesma letra minúscula na coluna não diferem entre si pelo teste de Tukey $(\mathrm{p} \leq 0,05 \%)$.

\section{Período de incubação}

O maior período de incubação dos ovos foi verificado em Eucalyptus saligna e Eucalyptus badjensis, com as primeiras eclosões ocorrendo no sétimo dia, enquanto que o menor período foi registrado em Eucalyptus dunnii, com início no quarto dia após a postura, indicando que esta espécie foi a mais adequada para o desenvolvimento dos insetos. O pico de eclosão das ninfas variou entre o quinto e sétimo dia, para a maioria das espécies (Tabela 1).

\section{Longevidade}

A longevidade média de Thaumastocoris peregrinus, incluindo machos e fêmeas, variou entre seis e 23 dias sendo maior em Eucalyptus dunnii, diferindo significativamente das demais espécies (Tabela 2). Em Eucalyptus globulus não foi registrado nenhum adulto.

A longevidade das fêmeas foi maior em Eucalyptus dunnii, diferindo dos demais. Em Eucalyptus bicostata, das 25 repetições, nenhuma fêmea chegou à fase adulta, apenas uma pequena quantidade de machos se tornaram adultos. De acordo com Noack e Rose (2007) e Crosa (2008), a longevidade média das fêmeas é de 30 dias, sendo superior àquela encontrada nesse estudo. Essa redução da longevidade de adultos pode ser uma resposta em relação às espécies de eucalipto, as quais reagem de forma diferenciada frente ao ataque do percevejo, e também pelo estudo ter sido feito em condições controladas, o que no campo poderia ser diferente. 
TABELA 2: Longevidade de fêmeas e machos de Thaumastocoris peregrinus (Hemiptera: Thaumastocoridae) criados em diferentes espécies de eucalipto (Temperatura $26 \pm 2^{\circ} \mathrm{C}$, UR de $60 \pm 10 \%$ e escotofase de 11 horas), Pelotas - RS, 2011. (Média seguida do erro padrão).

TABLE 2: Longevity of females and males of Thaumastocoris peregrinus (Hemiptera: Thaumastocoridae) reared on different species of eucalyptus (temperature $26 \pm 2^{\circ} \mathrm{C}, \mathrm{RH} 60 \pm 10 \%$ and scotophase 11 hours), Pelotas, RS state, 2011. (Average followed by the standard error).

\begin{tabular}{|c|c|c|c|c|}
\hline \multirow{2}{*}{ Espécie } & \multicolumn{4}{|c|}{ Longevidade (dias) } \\
\hline & Fêmea & $\mathrm{n}$ & Macho & $\mathrm{n}$ \\
\hline Eucalyptus badjensis & $06,69 \pm 0,29 \mathrm{c}$ & 13 & $6,75 \pm 0,41 \mathrm{c}$ & 8 \\
\hline Eucalyptus benthamii & $08,37 \pm 0,78 \mathrm{c}$ & 8 & $10,29 \pm 0,51 \mathrm{bc}$ & 14 \\
\hline Eucalyptus bicostata & - & _- & $14,5 \pm 0,29 \mathrm{~b}$ & 4 \\
\hline Eucalyptus dorrigoensis & $06,20 \pm 0,76 \mathrm{c}$ & $\overline{10}$ & $10,5 \pm 1,07 \mathrm{bc}$ & 10 \\
\hline Eucalyptus dunnii & $22,10 \pm 0,50 \mathrm{a}$ & 10 & $23,17 \pm 0,44 \mathrm{a}$ & 12 \\
\hline Eucalyptus grandis & $09,00 \pm 2,00 \mathrm{bc}$ & 3 & $07,00 \pm 1,15 \mathrm{c}$ & 4 \\
\hline Eucalyptus saligna & $12,67 \pm 0,21 \mathrm{bc}$ & 6 & $12,63 \pm 0,18 \mathrm{bc}$ & 8 \\
\hline Eucalyptus smithii & $09,45 \pm 1,21 \mathrm{bc}$ & 11 & $12,25 \pm 1,55 \mathrm{bc}$ & 12 \\
\hline Eucalyptus viminalis & $15,11 \pm 3,14 \mathrm{~b}$ & 9 & $15,46 \pm 2,60 \mathrm{~b}$ & 13 \\
\hline
\end{tabular}

Médias seguidas pela mesma letra minúscula na coluna não diferem entre si pelo teste de Tukey ( $\mathrm{p} \leq 0,05 \%)$.

TABELA 3: Ciclo biológico de fêmeas e machos de Thaumastocoris peregrinus (Hemiptera: Thaumastocoridae) criados em diferentes espécies de eucalipto (Temperatura $26 \pm 2^{\circ} \mathrm{C}$, UR de $60 \pm 10 \%$ e escotofase de 11 horas), Pelotas - RS, 2011. (Média seguida do erro padrão).

TABLE 3: Total cycle for females and males of Thaumastocoris peregrinus (Hemiptera: Thaumastocoridae) reared on different eucalyptus species (temperature $26 \pm 2{ }^{\circ} \mathrm{C}$, RH $60 \pm 10 \%$ and scotophase 11 hours), Pelotas, RS state, 2011. (Average followed by the standard error).

\begin{tabular}{lcccc}
\hline \multirow{2}{*}{ Espécie } & \multicolumn{3}{c}{ Ciclo biológico (dias) } \\
\cline { 2 - 5 } & Fêmea & $\mathrm{N}$ & Macho & $\mathrm{n}$ \\
\hline Eucalyptus badjensis & $41,69 \pm 1,89 \mathrm{~b}$ & 13 & $30,75 \pm 0,45 \mathrm{~d}$ & 8 \\
Eucalyptus benthamii & $31,75 \pm 0,90 \mathrm{e}$ & 8 & $34,64 \pm 0,32 \mathrm{bcd}$ & 14 \\
Eucalyptus bicostata & - & - & $40,00 \pm 0,41 \mathrm{~b}$ & 4 \\
Eucalyptus dorrigoensis & $31,80 \pm 0,74 \mathrm{e}$ & 10 & $35,20 \pm 1,28 \mathrm{bcd}$ & 10 \\
Eucalyptus dunnii & $48,70 \pm 0,40 \mathrm{a}$ & 10 & $49,58 \pm 0,42 \mathrm{a}$ & 12 \\
Eucalyptus grandis & $35,33 \pm 2,33 \mathrm{cde}$ & 3 & $32,75 \pm 0,85 \mathrm{~cd}$ & 4 \\
Eucalyptus saligna & $37,50 \pm 0,34 \mathrm{bcd}$ & 6 & $37,38 \pm 0,38 \mathrm{bc}$ & 8 \\
Eucalyptus smithii & $34,18 \pm 1,03 \mathrm{ed}$ & 11 & $35,42 \pm 1,28 \mathrm{bcd}$ & 12 \\
Eucalyptus viminalis & $33,11 \pm 3,10 \mathrm{bc}$ & 9 & $31,62 \pm 1,90 \mathrm{bc}$ & 13 \\
Médias seguidas pela mesma letra minúscula na coluna não diferem entre si pelo teste de Tukey (p<0,05\%). &
\end{tabular}

Soliman et al. (2012) constataram que, em Eucalyptus grandis, a longevidade foi maior para machos do que para fêmeas, 39,67 e 31,14 dias, respectivamente, permanecendo superior ao tempo registrado para esta mesma espécie no presente estudo, sendo sete e nove dias, respectivamente. Segundo esses mesmos autores, os machos tendem a ser mais longevos do que as fêmeas e, dependendo das condições podem se reproduzir com as fêmeas emergidas da $2^{\mathrm{a}}$ geração. É possível que, no presente estudo, a longevidade tenha sido afetada também pela redução do período de pós-oviposição, ocasionada pelo efeito varietal, afetando o comportamento do inseto.

\section{Ciclo biológico (ovo-adulto)}

O ciclo biológico de Thaumastocoris peregrinus nas diferentes espécies de eucalipto variou entre 30,75 e 49,58 dias. A maior duração do ciclo para machos e fêmeas foi registrado em Eucalyptus dunnii, 49,58 
e 48,70 dias, respectivamente (Tabela 3). Soliman et al. (2012) constataram, em seus estudos, que o ciclo biológico de vida dos machos variou de 19 a 99 dias, em diferentes clones de eucalipto. Ainda, em Eucalyptus grandis, estes autores verificaram que o ciclo biológico dos machos e fêmeas foi superior aos resultados obtidos neste estudo. Essas diferenças podem ter ocorrido em função da região, pelas diferentes características do alimento, que podem estar relacionadas ao clima e, assim, o efeito varietal foi maior no presente estudo. Variações no comportamento dos insetos são passíveis de ocorrência, em função de qualquer tipo de variação induzida pelo ambiente, sem que mudanças genéticas sejam necessárias (VIA et al., 1995). Fry (1992) já havia relatado que variedades de plantas podem apresentar diferentes performances em ambientes distintos, assim como os insetos, que podem responder de diferentes modos às variações genotípicas de suas plantas hospedeiras.

Das espécies de eucalipto estudadas, ficou evidente que Eucalyptus badjensis juntamente com Eucalyptus bicostata e Eucalyptus globulus, do ponto de vista da resistência de plantas, apresentaramse promissoras, pois interferiram negativamente sobre Thaumastocoris peregrinus, apresentando possível ocorrência de antibiose provavelmente associada com antixenose. Além disso, Eucalyptus badjensis tem se mostrado uma espécie altamente potencial para plantios no Sul do Brasil, em função da alta taxa de crescimento e alta resistência a geadas (HIGA; HIGA; ALVES, 2002).

\section{CONCLUSÃO}

As espécies Eucalyptus bicostata e Eucalyptus globulus afetaram negativamente no desenvolvimento, oviposição e longevidade do percevejo-bronzeado-do-eucalipto.

Eucalyptus dunnii foi a espécie em que Thaumastocoris peregrinus apresentou a melhor relação para desenvolvimento, oviposição e longevidade, sendo, portanto, desaconselhável seu plantio em monocultura.

\section{AGRADECIMENTOS}

À Capes pela concessão da bolsa de estudos e à Universidade Federal de Pelotas. À Fibria Papel e Celulose e a empresa GPR Serviços Florestais pelo auxílio na condução dos estudos.

\section{REFERÊNCIAS}

BUTTON, G. Thaumastocoris peregrinus. Forest facts, Pietermaritzburg, 2007. Disponível em: <www. nctforest.com/showpage. asp?id=44\&contentid=423\&ca tid=24>. Acesso em: 14 abr. 2012.

CARPINTERO, D. L.; DELLAPÉ, P. M. A new species of Thaumastocoris Kirkaldy from Argentina (Heteroptera: Thaumastocoridae: Thaumastocorinae), Zootaxa, Auckland, n. 1228, p. 61-68, 2006.

COUTURIER, G. et al. Biology of Discocoris drakei (Hemiptera: Thaumastocoridae) on Oenocarpus mapora (Palmae). Florida Entomologist, Lutz, v. 85, n. 1, p. 261-266, 2002.

CROSA, G. M. Thaumastocoris peregrinus Carpintero \& Delappé (Heteroptera: Thaumastocoridae): new pest found in eucalyptus in Uruguay. IUFRO Recent Advances in Forest Entomology, Pretoria, 2008.

FRY, J. D. On the maintenance of genetic variation by disruptive selection among hosts in a phytophagous mite. Evolution, New York, v. 46, p. 540-550, 1992.

GOULD, F. Sustainability of transgenic insecticidal cultivars: Integrating pest genetics and ecology. Annual Review of Entomology, Palo Alto, v. 43, p. 701-726, 1998.

HIGA, R. C. V.; HIGA, A. R.; ALVES, E. C. Comportamento de progênies de Eucalyptus badjensis Beuzev. \& Welch em dois locais da região Sul do Brasil. Boletim pesquisa Florestal, Colombo, n. 5, p. 89-97, 2002. JACOBS, D. H.; NESER, S. Thaumastocoris australicus Kirkaldy (Heteroptera: Thaumastocoridae): a new insect arrival in South Africa, damaging to Eucalyptus trees: research in action. South African Journal of Science, Johannesburg, v. 101, n. 5, p. 233-236, 2005.

LAUDONIA, S.; SASSO, R. First record of the bronze bug, Thaumastocoris peregrinus Carpintero \& Dellapé (Heteroptera: Thaumastocoridae), a new exotic pest of Eucalyptus trees in Italy. Bulletin of 
Insectology, Bologna, v. 65, n. 1, p. 89-93, 2012.

NOACK, A.; ROSE, H. Life-history of Thaumastocoris peregrinus and Thaumastocoris sp. in the laboratory with some observations on behaviour. General and Applied Entomology, Camden, v. 36, p. 27-33, 2007. SAS INSTITUTE. User's guide: statistics, version 9.1. Cary: SAS Institute, 2002.

SOLIMAN, E. P. et al. Biology of Thaumastocoris peregrinus in different eucalyptus species and hybrids. Phytoparasitica, Tel Aviv, v. 40, p. 223-230, 2012.

VIA, S. et al. Adaptive phenotypic plasticity. Consensus and Controversy. Trends in Ecology \& Evolution, Maryland Heights, v. 10, p. 212-217, 1995.

WINGFIELD, M. J. et al. Eucalypt pests and diseases: growing threats to plantation productivity. Southern Forests, Johanesburg, v. 70, n. 2, p. 139-144, 2008. 